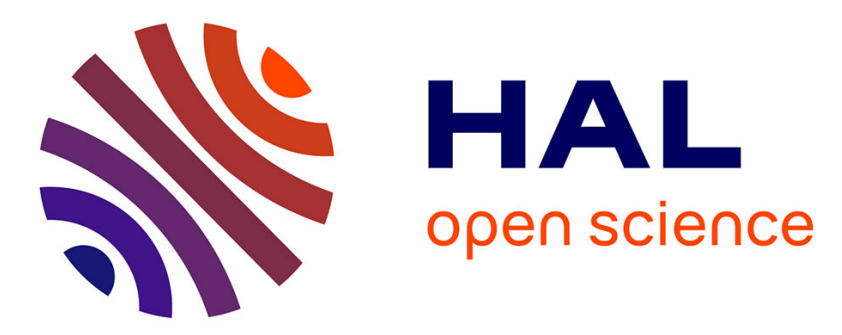

\title{
Spray-coated carbon nanotube carpets for creeping reduction of conducting polymer based artificial muscles
}

Aiva Simaite, Aude Delagarde, Bertrand Tondu, Philippe Souères, Emmanuel Flahaut, Christian Bergaud

\section{- To cite this version:}

Aiva Simaite, Aude Delagarde, Bertrand Tondu, Philippe Souères, Emmanuel Flahaut, et al.. Spraycoated carbon nanotube carpets for creeping reduction of conducting polymer based artificial muscles. Nanotechnology, 2017, 28 (2), pp.025502. 10.1088/0957-4484/28/2/025502 . hal-01413022

\author{
HAL Id: hal-01413022 \\ https://hal.laas.fr/hal-01413022
}

Submitted on 13 Dec 2016

HAL is a multi-disciplinary open access archive for the deposit and dissemination of scientific research documents, whether they are published or not. The documents may come from teaching and research institutions in France or abroad, or from public or private research centers.
L'archive ouverte pluridisciplinaire HAL, est destinée au dépôt et à la diffusion de documents scientifiques de niveau recherche, publiés ou non, émanant des établissements d'enseignement et de recherche français ou étrangers, des laboratoires publics ou privés. 


\title{
Spray-coated carbon nanotube carpets for creeping reduction of conducting polymer based artificial muscles
}

\author{
Aiva Simaite $^{\mathrm{e}, \mathrm{f}, *}$, Aude Delagarde ${ }^{\mathrm{e}, \mathrm{g}}$, Bertrand Tondu ${ }^{\mathrm{e}, \mathrm{f}}$, Philippe Souères $^{\mathrm{e}, \mathrm{h}}$, \\ Emmanuel Flahaut ${ }^{\mathrm{e}, \mathrm{g}}$, Christian Bergaud ${ }^{\mathrm{e}, \mathrm{h}, *}$ \\ ${ }^{a}$ CNRS, LAAS, 7 avenue du Colonel Roche, F-31400, Toulouse, France \\ ${ }^{b}$ Univ de Toulouse, INSA, LAAS, F-31400, Toulouse, France \\ ${ }^{c}$ Univ de Toulouse, Institut Carnot CIRIMAT, 118 route de Narbonne, 31062, Toulouse, \\ France \\ ${ }^{d}$ Univ de Toulouse, LAAS, F-31400, Toulouse, France
}

Abstract

During cyclic actuation conducting polymer based artificial muscles are often creeping from the initial movement range. One of the likely reasons of such behaviour is unbalanced charging during conducting polymer oxidation and reduction. To improve the actuation reversibility and subsequently the long time performance of ionic actuators, we suggest using spray-coated carbon nanotube $(\mathrm{CNT})$ carpets on the surface of the conducting polymer electrodes. We show that carbon nanotubes facilitate conducting polymer redox reaction and improve its reversibility. Consequently, in the long term, charge accumulation in the polymer film is avoided leading to a significantly improved lifetime performance during cycling actuation. To our knowledge, it is the first time a simple solution to an actuator creeping problem is suggested.

\footnotetext{
* Corresponding author

Email addresses: asimaite@iit.edu (Aiva Simaite), delagarde@laas.fr (Aude Delagarde), tondu@insa-toulouse.fr (Bertrand Tondu), tondu@insa-toulouse.fr (Philippe Souères), flahaut@chimie.ups-tlse.fr (Emmanuel Flahaut), bergaud@laas.fr (Christian Bergaud)
} 
Keywords: Carbon nanotubes, spray coating, conducting polymers, creep, actuators

\section{Introduction}

Ionic conducting polymer based actuators are one of many alternatives to traditional actuators. Due to a low actuation voltage, lightness and compliance conducting polymer based actuators are biocompatible and attractive for applications in biomedical engineering and biotechnology [1, 2]. Also, CPAs can induce a relatively large force, and only small currents are needed to hold constant strain at DC voltage $[2,3]$.

The use of conducting polymers as actuators is based on the linear dimensional changes during their electrochemical doping and dedoping process. There are several simultaneous and subsequent processes involved in changing the electrochemical state of the conjugated polymer, such as the charge and mass transport and the conformational changes of the polymer. These processes depend on the material properties, actuation and cycling conditions and affect the performance of the actuators regarding strain, speed, stress and efficiency in a complex and still not fully understood way [4]. For example macroscopic charge transport of intrinsically conductive polymer films requires 'hopping' between polymer chains. Because of this, the composition and the morphology of the polymer film might have a significant influence on the charge transfer $[5,6,7,8,9]$. Furthermore, volumetric expansion mechanism needs to take into account the doping of the conducting polymer, the size and mobility of the anion and cation in the electrolyte and the solvent $[10,11,12]$. Finally, even though the flux of ions to and from the mate- 
rial is considered to be the primary mechanism for producing deformation, conformational changes in the polymer could also play a role $[5,7,13]$.

Despite unique advantages that CPAs offer, redox reactions and polymer swelling are also the main drawbacks of the conducting polymer based devices [14]. In most cases, actuators fail gradually due to fatigue during the actuation or due to spontaneous self-degradation [15]. Gradual deterioration is observed and reported most often and could be caused by fatigue of the polymer, i.e. change in the mechanical properties, chemical degradation of the material, receding electrolyte or irreversible redox reactions and reduction in the charge transfer $[4,15,16,17,18,19 ?]$. The later is also one of the reasons of creeping $[16,20]$.

Creeping in CPAs is observed as a continuous displacement of the movement range [21]. It originates from the incomplete recovery of the initial position on consecutive actuation cycles. There are two identified reasons causing such behaviour: (1) cycling induced solvent swelling suggested by Melling et al. [20] and (2) unbalanced charging characterized by Kaneto et al. $[22,23,24]$. In the first case, cycling opens the polymer matrix so that with each cycle ions diffuse faster and therefore deeper into the film. Consequently, if present, more solvent can irreversibly accommodate in between polymer chains $[7,20,23,25]$. In the case of unbalanced charging, both, faster actuation rate during reduction [20] and oxidation [26] were previously observed. It is attributed to the higher electronic conductivity in the oxidised state and the higher ion conductivity because of the more expanded, open and swollen polymer. In any case, unbalanced charges at the end of an actuation cycle induce accumulation of the ions in the films [23]. 
Carbon nanotubes are widely used for applications in conducting polymer based actuators, primarily to increase the conductivity, robustness and stiffness of the polymer matrix [27, 28, 29]. Besides, incorporation of carbon nanomaterials in actuators was shown to increase the Faradaic capacitance of the conducting polymer, the electrical conductivity and to consequently increase actuators stroke and work per cycle [30, 31, 32, 33, 34]. Just as in batteries, incorporation of the CNTs also increases the redox reversibility and significantly reduces creeping [35, 27, 32]. Nevertheless, one of the biggest challenges for using these composites is the low solubility of carbon materials [36]. Only small amounts of the more conductive, non-oxidised carbon nanotubes or graphene can be homogeneously dispersed in the polymer solutions [37, 38]. Higher concentrations lead to the formation of agglomerates. When incorporated during the electropolymerization [33], the concentration of carbon nanotubes is difficult to control. Other fabrication methods, i.e. layer-by-layer deposition [34], drop casting [32], soaking [31], that would allow to increase the CNTs amount in the polymer actuators were suggested, but they also require soluble and easily processable CNTs.

In this article, we suggest using spray coated carbon nanotube carpets (CNTCs) to reduce creeping and improve the long-time performance of the actuators. We show that spray-coating allows deposition of large quantities of carbon nanotubes (CNTs) leading to well connected and conductive networks. Furthermore, CNTC is deposited only on the surface and does not create a layered structure that is prompt to de-lamination. Finally, we show that CNT carpets increase Faradaic capacitance of conducting polymer electrodes, improve charge-discharge reversibility and subsequently lead to a 
significant decrease in creeping. CNTCs effects are particularly advantageous if actuators are needed for cyclic positioning.

\section{Materials and Methods}

\subsection{Materials}

Hydrophobic, polyvinylidene fluoride (PVDF) membranes with a mean pore diameter of $0.1 \mu \mathrm{m}$ were bought from Merck Millipore Corp (Millipore VVHP04700 Durapore Membrane Filter, thickness - $120 \mu \mathrm{m}$ and $47 \mathrm{~mm}$ in diameter). Poly(3,4-ethylenedioxythiophene) polystyrene sulfonate (PEDOT:PSS) $1.3 w t \%$ conductivity grade dispersion in water (483095 Aldrich), with a PEDOT:PSS ratio of 1:2.5 was bought from Sigma-Aldrich. The ionic liquid emimTFSI was bought from Solvionics (Im0208a).

Double-walled carbon nanotubes (CNTs) were synthesized in CirimatCNRS as reported in [39] and provided in water sodium dodecyl sulfate (SDS) suspension. CNTs samples contained $77 \%$ of double-walled CNTs, $18 \%$ of single-walled CNTs and $5 \%$ of triple-walled CNTs that have a length ranging between 1 and $10 \mu \mathrm{m}$ and a diameter of $1.2-3.2 \mathrm{~nm}$. They were

shown to exhibit a metallic electrical behaviour [40]. $0.1 w t \%$ of CNTs and $1 w t \%$ of SDS were dispersed in deionized water and separated using $150 \mathrm{~W}$ ultrasound (VibraCell 75042, Bioblock Scientific) in an ice bath for 30 min. The suspension was then centrifuged (16000 rpm, $10 \mathrm{~min}$ ) and the supernatant was collected. Before spray coating, all the suspensions were dispersed using pulsed ultrasound (5 sec on and $5 \mathrm{sec}$ off) for $10 \mathrm{~min}$ at $150 \mathrm{~W}$. For spraying SDS, $1 w t \%$ solution was prepared in deionized water. 


\subsection{Fabrication of PEDOT:PSS/PVDF/PEDOT:PSS actuators}

Figure 1 depicts a schematic illustration of materials, as well as the fabrication process of actuators used in this work. We were using PEDOT:PSS/PVDF/ionic liquid actuators made by solvent casting as previously reported and characterized by our group [41, 42]. Nevertheless, carbon nanotubes could equally enhance actuators produced in other ways or based on other conducting polymers.

Hybrid PVDF/PVDF-graft-PEGMA membranes were prepared to ensure excellent adhesion between solvent cast conducting polymer film and the membrane [41]. Grafting of about $0.6 \mathrm{mg}$ of PEGMA on each side of PVDF membrane leads to $30 \mu m$ PVDF-graft-PEGMA layer (hydrophilic) on each surface and $60 \mu \mathrm{m}$ of hydrophobic PVDF membrane in the center. For the simplicity, in the article, PVDF/PVDF-graft-PEGMA membranes are referred as PVDF. The electrical conductivity of PEDOT:PSS was enhanced by secondary doping with polyethylene glycol (PEG) with average $M n-400$ (PEG400) [43]. 1 vol\% of PEG400 in PEDOT:PSS solution were mixed on a mechanical shaker for 3 hours at $300 \mathrm{rpm}$ and if needed kept at $4{ }^{\circ} \mathrm{C}$ before use. Further in the article PEDOT:PSS secondary doped with PEG is called PEDOT:PSS.

For the fabrication of actuators, functionalized PVDF membranes were fixed in a $\varnothing 45 \mathrm{~mm}$ aluminium clamp and $3 \mathrm{ml}$ of PEDOT:PSS, were solventcast. Membranes were dried in air in a UV-reduced environment for at least 20 hours. The other side of the membrane was processed in the same way. Membranes were thermally annealed in the oven for 1 hour at $70{ }^{\circ} \mathrm{C}$. In order to create a trilayer, the procedure was repeated on the other side of the 

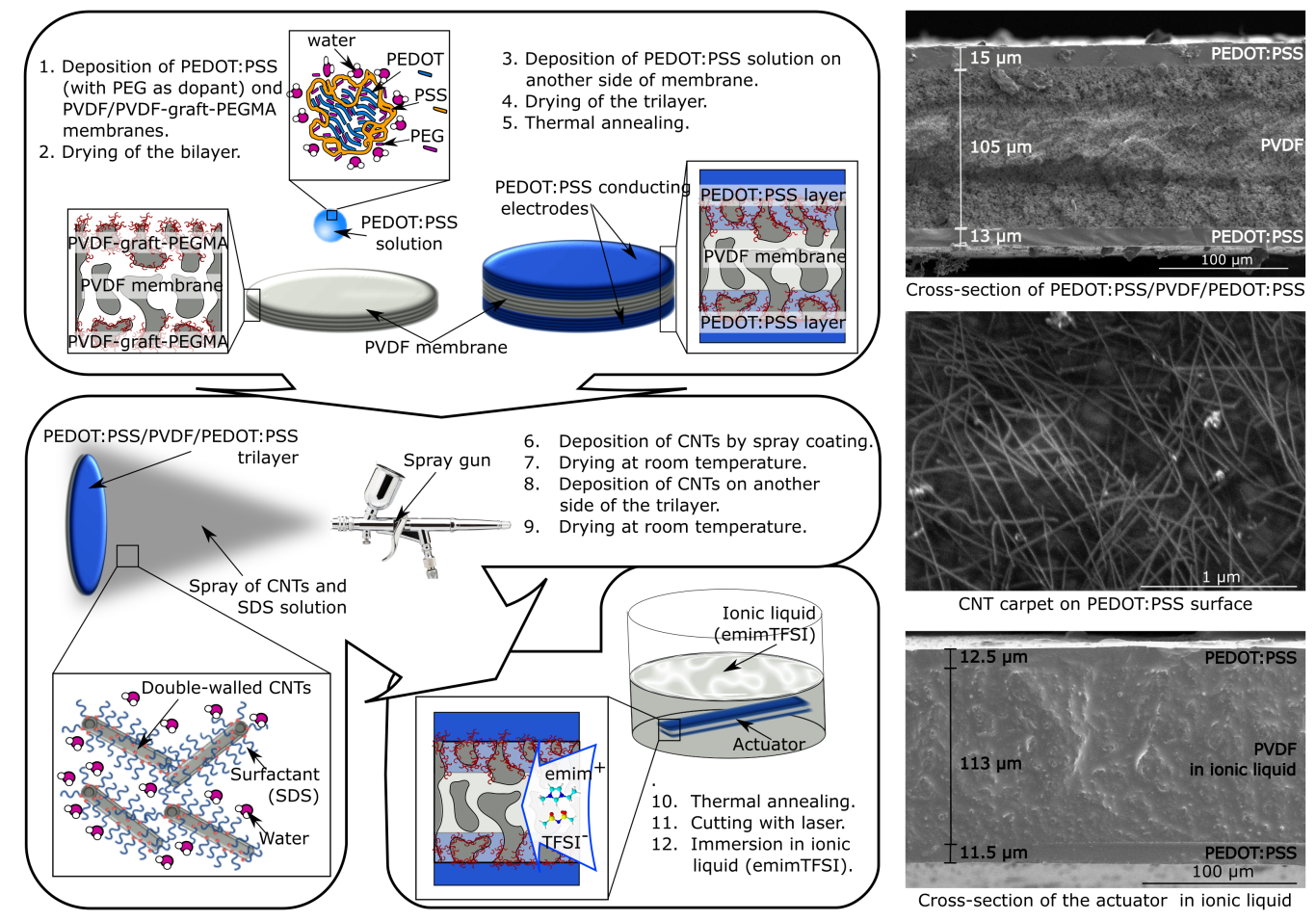

CNT carpet on PEDOT:PSS surface

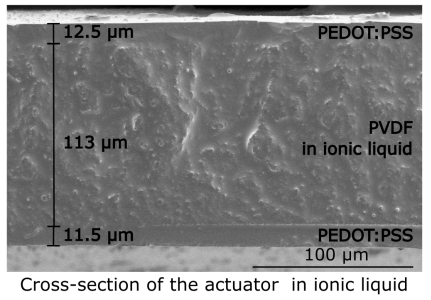

Figure 1: Illustration of the main fabrication steps of PEDOT:PSS/PVDF/PEDOT:PSS actuators with sprayed carbon nanotube carpets. Insets show assumed morphology of PEDOT:PSS secondary doped with PEG structure in solution, PVDF/PVDF-graftPEGMA/PVDF hybrid membranes (produced as in [41]), CNT and SDS organisation in aqueous solution and molecular structure of the ionic liquid. SEM images of the crosssections of the actuators on the right show the structure of the actuators in dry state, after immersion in ionic liquid and the surface of PEDOT:PSS with sprayed CNT carpet (CNTC). 
Table 1: Geometry and electrical properties of actuators

\begin{tabular}{|c|c|c|c|c|c|}
\hline & $\mathrm{h}_{d r y}{ }^{a}, \mu m$ & $\mathrm{~h}_{e l}{ }^{b}, \mu m$ & $\mathrm{w}_{d r y}{ }^{c}, m g$ & $\mathrm{w}_{I L s}{ }^{d}, m g$ & $\mathrm{R}_{e l}{ }^{e}, k \Omega$ \\
\hline without CNT & 133 & $15 / 13$ & $3.6 \pm 0.1$ & $5.8 \pm 0.2$ & $5.1 \pm 3.7$ \\
with CNT & 142 & $20 / 10$ & $3.5 \pm 0.2$ & $6.2 \pm 0.3$ & $1.6 \pm 0.5$ \\
with SDS & 125 & $10 / 9.5$ & $3.1 \pm 0.3$ & $5.5 \pm 0.3$ & $1.5 \pm 0.5$ \\
\hline
\end{tabular}

${ }^{a}$ Thickness of dry trilayer; ${ }^{b}$ PEDOT:PSS electrode thickness; ${ }^{c}$ Weight of dry actuator; ${ }^{d}$ Weight of the actuator with ionic liquid; ${ }^{e}$ Resistance of the electrodes.

membrane. Dried actuators were composed of $15 \mu m$ thick PEDOT:PSS electrodes, 20-25 $\mu \mathrm{m}$ interfacial layer and $60 \mu \mathrm{m}$ insulating PVDF membrane as shown in the scanning electron microscope (SEM) image on the top right of Figure 1. SEM images were obtained using a Hitachi $S-4800$ field emission scanning electron microscope (acceleration voltage $800 \mathrm{~V}$, working distance of about $5 \mathrm{~mm}$ ). Table 1 summarizes the geometry and other properties of actuators used in this work.

\subsection{Spray coating of CNTs}

For post-treatment of actuators with CNTs or SDS, the membrane cast with conducting polymer on both sides was divided into 3 parts. One part was not modified and was used as a reference. The second part was used for deposition of CNTs and the third part was spray coated with only surfactant (SDS) solution. Spray coating was done under a fume hood and in a glovebox (Captair Pyramid Glovebox). PEDOT:PSS/PVDF/PEDOT:PSS trilayers were stick to the glass slide, next to two silver electrodes and a piece of 
paper used as a color indicator as shown in Figure S.1. The following spraying parameters were used: nozzle of $0.2 \mathrm{~mm}$ and associated needle, pressure $1.5 \mathrm{~kg} f / \mathrm{m}^{2}$, valve opening - 1.5 turn, distance - about $30 \mathrm{~cm}$. To ensure homogeneous spraying, the spray was targeted by a laser pointed. CNTs were sprayed until the resistance between silver electrodes decreased to about $10 k \Omega$ corresponding to approximately $6 \mathrm{ml}$ of CNTs suspension. A dense and well interconnected CNT carpet was formed as shown in the SEM picture of the surface of PEDOT:PSS in Figure 1. After spraying on one side, the membrane was dried under the fume hood at room temperature for $1-2$ hours to make sure the surface of the trilayer is dry. The other side of the trilayer was treated in the same way. For post-treatment with a surfactant, $6 \mathrm{ml}$ of SDS solution in water were sprayed. After the spray of CNTs or SDS solutions, trilayer was again dried in an oven for 1 hour at $70{ }^{\circ} \mathrm{C}$.

\subsection{Geometry of PEDOT:PSS/PVDF/PEDOT:PSS actuators}

$15 \times 2 \mathrm{~mm}^{2}$ rectangles were cut by $\mathrm{CO}_{2}$ laser $(45 \mathrm{~W}$ power, $100 \mathrm{~cm} / \mathrm{s}$ speed, $1000 \mathrm{~Hz}$, Trotec FineMarker Hybrid) and incubated in ionic liquid for 24 hours before use. Each actuator was then weighted and its thickness measured by digimatic indicator (Mitutoyo Absolute). They were kept in ionic liquid (emimTFSI) for 24 hours before characterisation and then in a closed tube if not used immediately. After soaking, their weight and thickness were measured again and is summarized in Table 1. Just as reported previously by our group [41], incubation in ionic liquid slightly decreases the thickness of PEDOT:PSS to about $12 \mu \mathrm{m}$ leading to an overall actuator thickness of $137 \mu \mathrm{m}$.

Actuators with carbon nanotubes might also influence the amount of ionic 
liquid adsorbed during the same time of incubation $(2.7 \mathrm{mg}$ and $2.2 \mathrm{mg}$ with and without CNTs respectively). With ionic liquids, CNTs form gel-like composites (bucky gels) meaning that in addition to storing the ionic liquid in PVDF membrane, some of it could also be adsorbed by CNT carpet on the surface of PEDOT:PSS. If that is the case, it could have interesting implications on redox performance of PEDOT:PSS itself, as counter ions would not need to diffuse through the PEDOT:PSS film to balance the charge. Instead, ions adsorbed by CNTs could participate in a redox reaction. This possibility is not discussed further in the article and requires further investigation.

\subsection{Electro-chemo-mechanical characterization of PEDOT:PSS/PVDF/PEDOT:PSS actuators}

Resistance and conductivity of PEDOT:PSS were measured between two extremities of each electrode and between two electrodes, by applying 0.1 $0.5 V$ voltage (with $0.01 V$ increment) and measuring current, using a Suss PA200 probe station and an Agilent 4142B tester. Conductivity was then calculated as: $\sigma=\frac{L}{R * W * h}$, where $R$ is the measured resistance, $L, W$ and $h$ are length $(15 \mathrm{~mm})$, width $(2 \mathrm{~mm})$ and thickness $(20 \mu \mathrm{m})$ of the rectangle. We used thickness measured by SEM and did not include PEDOT:PSS infiltrated in the membrane.

Cyclic voltammetry (CV) measurements were performed using an Autolab potentiostat PGSTAT30 (Metrohm Autolab) coupled with NOVA 1.8 Software at room temperature $\left(23 \pm 1^{\circ} \mathrm{C}\right)$. A two-electrode configuration was adopted (equivalent to the one used for bending characterization), in which the two surfaces of PEDOT:PSS were working and reference electrodes. CV measurements were performed at various voltages and scan rates. The spe- 
cific capacitance was calculated as $C=\frac{\int i v \mathrm{~d} v}{2 \mu m \Delta V}$, where $i, v$ are measured current and applied potential, $\mu, m$ and $\Delta V$ are the scan rate, the mass of active materials and the potential window respectively. Electrochemical impedance spectroscopy (EIS) measurements were performed with the same equipment and in the actuator working configuration. The magnitude of the alternative signal was $10 \mathrm{mV}$ and the measurements were done in a frequency range between $100 \mathrm{kHz}$ and $10 \mathrm{mHz}$.

Actuator bending was tracked by laser displacement sensor (optoNCDT 1302, MicroEpsilon) at a position of about $2 \mathrm{~mm}$ from the end of the actuator. Scheme of the set-up used for actuation and tracking is shown in Figure 2A. All measurements were performed at room temperature and humidity that were slightly varying. A laser displacement sensor (optoNCDT 1700, MicroEpsilon) was connected to Ni-DAQmx for data logging and was also used for signal generation and current measurements. For creep measurements, displacement measured by laser was used as a feedback to switch actuators position to $1 \mathrm{~mm}$ above or below the initial by changing the polarity of the applied voltage $( \pm 1.5 \mathrm{~V})$. The time needed to reach the set position was calculated for each half cycle.

\section{Results and Discussion}

\subsection{Harmonic response}

A continuous displacement of the movement range, i.e. creeping, can be most easily observed during harmonic actuation as shown in Figure S.2 (Supporting information). Actuation starts with cycling in between about $+1.2 \mathrm{~mm}$ and $-1 \mathrm{~mm}$ and after 2 minutes or 25 cycles, the position shifts to 
A

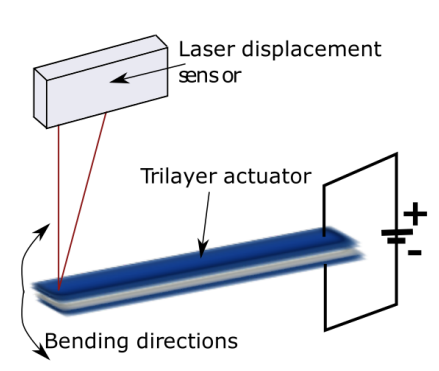

B

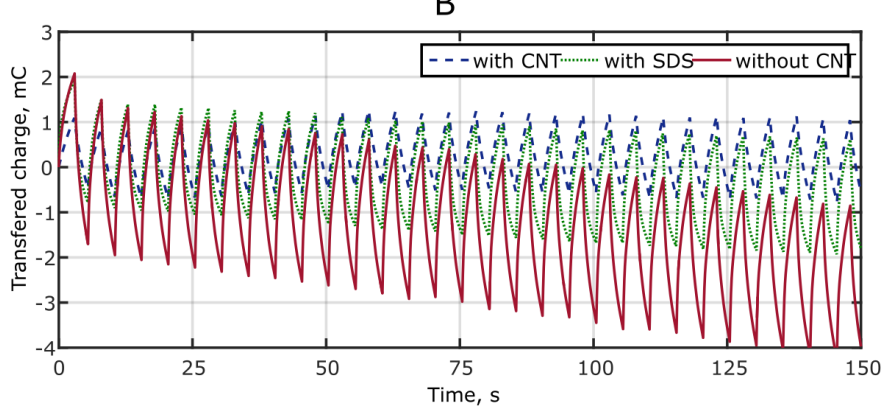

Figure 2: a) Schematic illustration of the set-up used for actuation characterization by laser displacement sensor. B) Calculated transferred charge during actuation of trilayer actuators with and without post-treatments. $1.5 \mathrm{~V}$ square wave was used for actuation.

$+1 \mathrm{~mm}$ and $-1.2 \mathrm{~mm}$. Because solvent was not used for fabrication of our actuators, the most likely reason of creeping is unbalanced charging [22, 23, 24]. Transferred charge during harmonic actuation is shown in Figure 5B. It was calculated as integrated current transferred during actuation. In the case of pristine PEDOT:PSS without CNTC significant and fast creeping is observed. In less than $150 s$ one side of the PEDOT:PSS film continues being charged but is not completely discharged. That shows that, indeed, charges are being accumulated and stored on one side of the trilayer.

On the other hand, actuators sprayed with CNTs work with lower displacement amplitude, but its position remains relatively stable (Figure S.2). As shown in Figure 2B harmonic response of the transferred charge for actuators with CNTCs is significantly more reversible and remains in the initial range $( \pm 1 m C)$. Even though, less significant, improvement in charging reversibility is also noticeable for actuators with sprayed SDS. After $150 \mathrm{~s}$ of actuation charging and discharging remains in between about $0.8 \mathrm{mC}$ and 
$-2 m C$.

The morphology of PEDOT:PSS and its compact structure might be one of the reasons of irreversible expansion and charge accumulation, as previously suggested by Melling et al. [20]. Harmonic cycling opens the polymer matrix so that with each cycle ions can diffuse faster and therefore deeper into the film. When polarity is reversed, some of the ions might remain there. In this case, spraying with a surfactant such as SDS could improve the reversibility of cycling by opening compact PEDOT:PSS structure and subsequently facilitate ion transport through it.

Nevertheless, CNTCs show much more significant improvement in charge transfer reversibility. To better understand this actuation behaviour, various electro-chemo-mechanical experiments were performed.

\subsection{Bending characterization}

Actuator performance was evaluated by tracking the displacement at its tip with the laser displacement sensor. The peak-to-peak displacement versus actuation frequency is shown in Figure 3A. Measurements were done by applying square voltage wave with the amplitude of $1.5 \mathrm{~V}$ and decreasing frequency of the wave after several cycles from $5 \mathrm{~Hz}$ to $10 \mathrm{mHz}$. At $1.5 \mathrm{~V}$ the performance of carbon nanotube actuators was poorer in comparison to simple actuators. For instance, at low frequencies, $10 \mathrm{mHz}$ actuator without CNTCs produce $1.5 \mathrm{~mm}$ larger displacement amplitude. Results are surprising because most of the previously reported composites with carbon nanomaterials were improving the performance of actuators at all frequencies $[44,45,34]$. Furthermore, CNTCs also reduce the electrical resistance of PEDOT:PSS electrodes from $5.1 \pm 3.7 k \Omega$ to $1.6 \pm 0.5 k \Omega$. We think that 
the increased stiffness of the actuators that was not measured in this study is the most likely explanation for a decrease in their response time [34].

Figure $3 \mathrm{~B}$ and $3 \mathrm{C}$ show the comparison of the performance of the actuators without and with CNTC (respectively) at voltages in the range from $0.5 \mathrm{~V}$ to $2.0 \mathrm{~V}$. In the case of actuators without CNTC, at low voltages below $1 \mathrm{~V}$ actuators were reaching a nearly stable position in less than $5 \mathrm{~s}$. This is a consistent response of actuators working due to the charging of a double layer capacitance at the interface between the membrane and PEDOT:PSS as suggested by Okuzaki et al. [46]. Only above $1.5 \mathrm{~V}$ PEDOT:PSS is starting to be reduced causing the expansion and bending due to the ion incorporation in the polymer film. This can be seen in Figure 3B, where slow continuous bending is shown even after $40 s$ of actuation. Furthermore, displacement of the actuator tip is less sensitive to the voltage change above $1 \mathrm{~V}$. Results suggest two possibilities: (i) ionic conductivity of PEDOT:PSS is very low (ions do not move though the film), (ii) charge transfer in PEDOT:PSS is limited.

Different responses to voltage are observed for actuators with CNTCs as shown in Figure 3C. For voltages lower than $1.5 \mathrm{~V}$ actuator responds slower and reaches slightly lower plateau value. That is, most likely, a consequence of an increased stiffness of the actuator [34]. Nevertheless, CNTCs seem to significantly improve the performance of the actuators when voltages are higher than $1.5 \mathrm{~V}$. At $2 \mathrm{~V}$ a displacement of the actuator was significantly larger, $2.6 \mathrm{~mm}$ versus $3.2 \mathrm{~mm}$ in $50 \mathrm{~s}$ without and with CNTCs respectively, and seemed to be further increasing. This is an indication that carbon nanotubes positively affect the redox behaviour of PEDOT:PSS. Furthermore, 

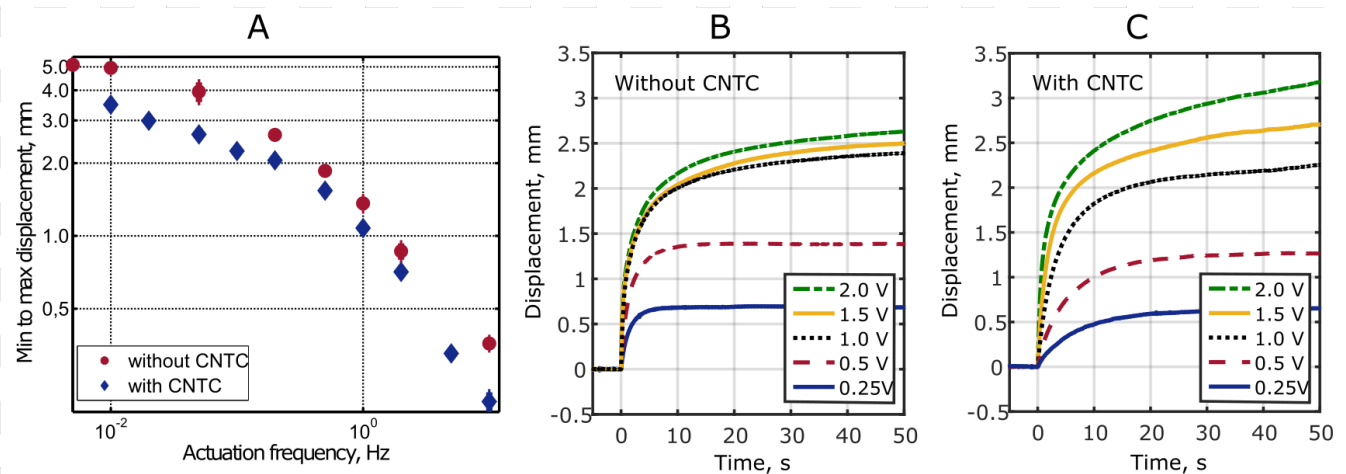

Figure 3: A) Frequency response of actuators with and without carbon nanotubes. Calculated after actuation with $1.5 \mathrm{~V}$ square wave as an average of at least 3 cycles. B) Open loop step response of actuators without CNTC at different voltages. C) Open loop step response of actuators with CNTC at different voltages.

the displacement of the actuators remains sensitive to the higher applied voltages $(3.3 \mathrm{~mm}$ and $2.3 \mathrm{~mm}$ after $50 \mathrm{~s}$ for $2 \mathrm{~V}$ and $1 \mathrm{~V}$ respectively). Results suggest that CNTCs also facilitate the ion transport to the film.

\subsection{Electrochemical characterization}

In order to further explore electrochemical properties of actuators, cyclic voltammetry $(\mathrm{CV})$ measurements were performed in an actuator working configuration and are shown in Figure 4A. As expected from previous results, redox behaviour was noticeable for voltages higher than $1 \mathrm{~V}$. Actuators with CNTC show a significantly larger increase in current at higher voltages confirming their influence in improving redox. The specific capacitance of the actuators is at least $25 \%$ higher when CNTCs are used (shown in Figure S.3 (Supporting Information)). Furthermore, the shape of oxidation and reduction peaks is also more similar when CNTCs are used. This suggests improvement in reversibility of the redox reaction. As a reference 

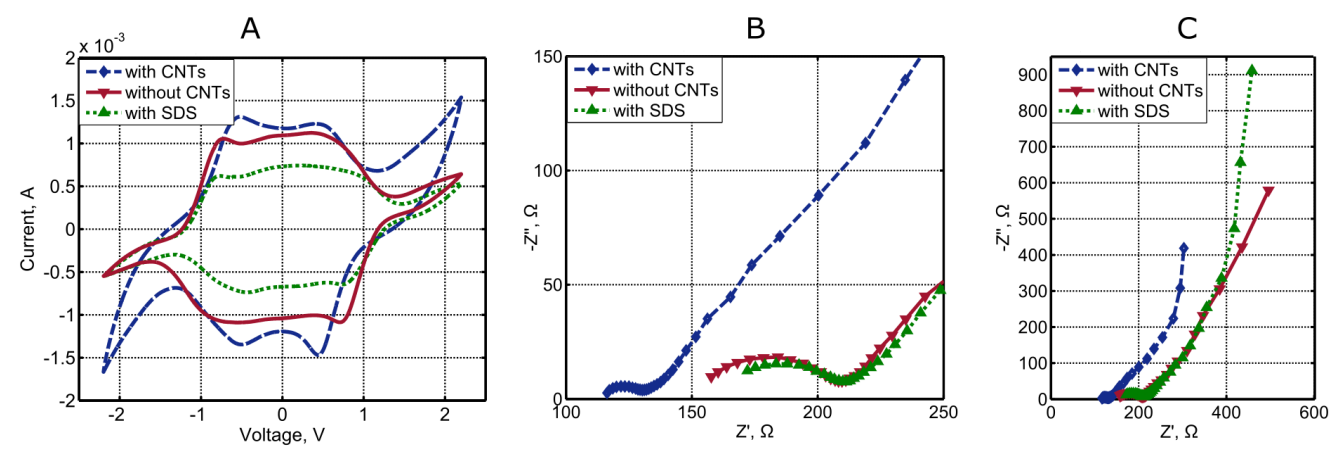

Figure 4: A) CV voltammogram of actuators without CNTC (red full line), with CNTC (blue dashed line) and with SDS (green dotted line) $(20 \mathrm{mV} / \mathrm{s}$ scan rate). E-F) Nyquist plots for PEDOT:PSS with and without CNTC measured without prior bias potential from $100 \mathrm{kHz}$ to $10 \mathrm{mHz}$.

CV measurements were also performed for actuators with only surfactant (SDS) sprayed on the surface. Neither does SDS alter the specific capacitance of the actuator nor show any improvement to redox behaviour. These CV responses imply that carbon nanotubes have an influence on improving the redox activity of the conducting polymer based actuators and its reaction reversibility.

As previously mentioned, in addition to redox behaviour, cycling reversibility and creeping could also be influenced by the morphology of PEDOT:PSS film. In order to evaluate ionic conductivity of PEDOT:PSS films with and without CNTC, electrochemical impedance spectroscopy (EIS) was performed at the actuator working configuration with the potential amplitude of $\mathrm{AC}$ kept at $10 \mathrm{mV}$ and in the frequency range from $100 \mathrm{kHz}$ to $10 \mathrm{mHz}$. Results presented as a Nyquist plot are shown in Figure 4B-C. Quantitative data fitting and extraction of conductivities were unsuccessful 
due to the complex quasi-trilayer structure of the actuator [41]. Nevertheless, actuators with CNT carpets had lower resistance at high frequency (in a range between $110 \Omega$ and $140 \Omega$ ). Our results suggest that PEDOT:PSS electrode with CNTC has a higher combined ionic and electronic conductivity.

We have already mentioned, that CNTCs reduce the resistance of PEDOT:PSS electrodes from $5.1 \pm 3.7 k \Omega$ to $1.6 \pm 0.5 k \Omega$. On the other hand spraying PEDOT:PSS with only SDS solution also decrease the resistivity of the film to $1.5 \pm 0.4 k \Omega$. It was previously reported that SDS improve the electrical conductivity of PEDOT:PSS by altering its PEDOT:PSS towards a more swollen and less compact core/shell structure [47]. We think, that less dense morphology also improve the ionic conductivity as it allows easier ion penetration and movement through the PEDOT:PSS film [20]. On the other hand, the difference in EIS response between pristine PEDOT:PSS and PEDOT:PSS with SDS is not significant. That says that even though SDS might play a role in increasing the overall conductivity of actuators, CNTC, i.e. improvement in redox reaction of PEDOT:PSS, is significantly more important.

\subsection{Creeping reduction}

To sum up, we have demonstrated that CNTCs significantly improve redox behaviour of PEDOT:PSS and its overall electrical and ionic conductivity. Both effects are known to contribute to cycling reversibility and could explain reduced charge accumulation shown in Figure 2B. To demonstrate the importance of reversible cycling on the long term performance of conducting polymer actuators we actuated CPAs with and without CNTC using 

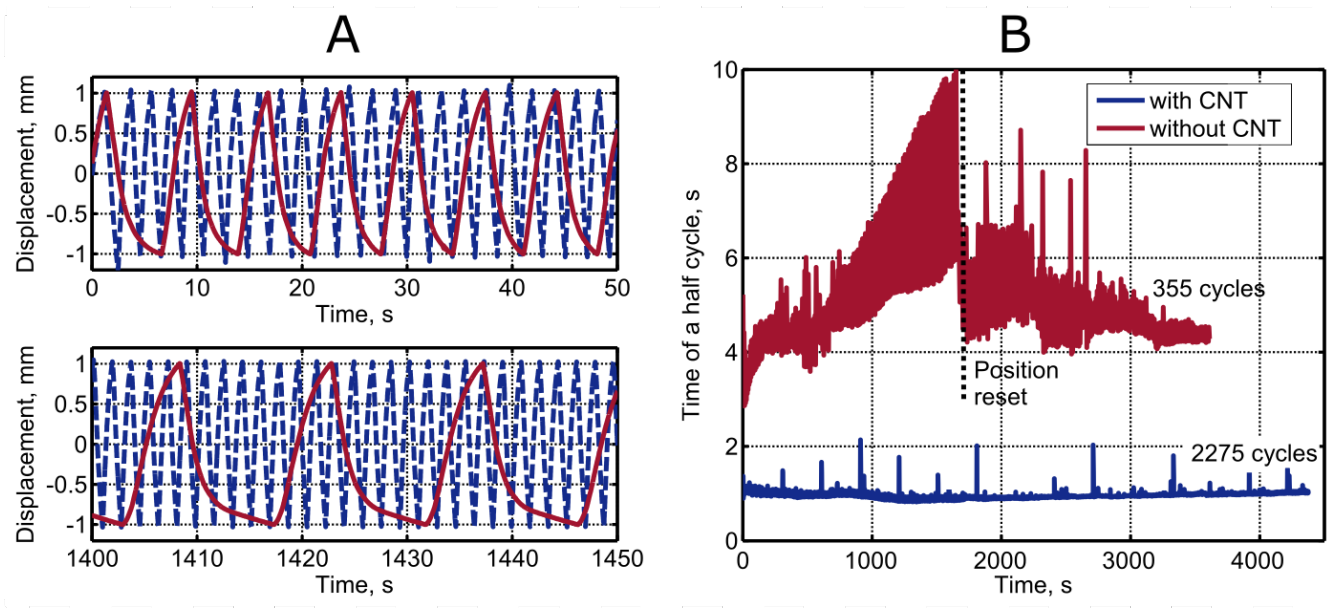

Figure 5: A) Bending profiles vs time for actuators with (blue dotted line) and without (red line) CNTC. The voltage polarity $( \pm 1.5 \mathrm{~V})$ was changed every time the actuator reached the $\pm 1 \mathrm{~mm}$ set value. B) Time needed for the tip of the actuator to move from to $\pm 1 \mathrm{~mm}$ from its initial position vs time for the CNTs (blue) and simple (red) actuators. $\pm 1.5 \mathrm{~V}$ was used to change the direction of bending as soon as the actuator tip reached the set value. For actuator without CNTC the value was reset to $-0.8 \mathrm{~mm}$ and $+1.2-\mathrm{mm}$ due to the large creeping (shown as dotted line).

feedback from laser displacement sensor (creeping during harmonic cycling eventually leads to actuation out of the laser measurement range). Actuation asymmetry and creep were estimated by comparing the time needed for the actuator to do a half cycle, i.e. to bend from one extremum to another. The voltage polarity $( \pm 1.5 \mathrm{~V})$ was changed every time the actuator got to the set $\pm 1 \mathrm{~mm}$ value. The time needed for the actuator to reach these positions was then extracted and is plotted versus actuation time in Figure 5A.

In the case of the actuator with no CNTC (full red line in Figure 5A), the time needed for the tip of the actuator to move one direction is near 5 times longer than the other. This time difference is further increasing and after 
$1400 s$ it takes $10 s$ for the actuator to reach the lower extreme. The difference in time needed to move the actuator upwards or downwards can also be clearly seen in Figure 5B as a filled area. After less than $2000 s$ the position of actuator without CNTC was reset as it was not reaching the lower limit of $-1 \mathrm{~mm}$. On the other hand, actuators with CNTC demonstrated nearly the same time for each half-cycle, and it remained rather constant for more than 2200 cycles or 1 hour of actuation (dashed blue line in Figure 5A-5B). This remarkable improvement in actuator performance is in agreement with our previous discussion - CNT carpets increased redox reaction reversibility that consequently reduced charge accumulation in the PEDOT:PSS film leading to reduced creeping of the actuator.

\section{Conclusions}

We have demonstrated that carbon nanotube carpets deposited by spray coating could be used to improve conducting polymer based artificial muscles. We suggest that CNTC increases the redox activity of PEDOT:PSS leading to better performance at voltages above $1.5 \mathrm{~V}$. Furthermore, together with an ionic surfactant, i.e. SDS, CNTs increase overall electrical and ionic conductivity of PEDOT:PSS, consequently, improves the redox cycling reversibility and reduce the charge accumulation in the polymer film. These effects lead to a significantly reduced electrochemical creeping of conducting polymer based actuators. It is the first time a method for limiting electrochemical creeping of conducting polymer based actuators is suggested. As creeping is one of the main limitations of conducting polymer based devices, this could be a significant step forward towards their commercial applications. Finally, 
spray coating is a simple and a versatile technique that can be used as an additional step after any other conducting polymer deposition.

\section{Acknowledgement}

The authors acknowledge the French Ministry of Defence, The Armaments Procurement Agency (DGA) for the Ph.D. grant for work on Development of electroactive polymer based actuators for actuation in robotics. This work was partly supported by the French RENATECH network.

\section{References}

[1] P. G. Madden, J. D. Madden, P. Anquetil, N. Vandesteeg, I. W. Hunter, The relation of conducting polymer actuator material properties to performance, Oceanic Engineering, IEEE Journal of 29 (3) (2004) 696-705.

[2] E. Smela, Conjugated polymer actuators for biomedical applications, Otero 7 (2003) 22.

[3] E. W. Jager, N. Masurkar, N. F. Nworah, B. Gaihre, G. Alici, G. M. Spinks, Patterning and electrical interfacing of individually controllable conducting polymer microactuators, Sensors and Actuators B: Chemical 183 (2013) 283-289.

[4] A. Simaite, Development of ionic electroactive actuators with improved interfacial adhesion: towards the fabrication of inkjet printable artificial muscles, Ph.D. thesis, INSA Toulouse (2015). 
[5] X. Chen, K.-Z. Xing, O. Inganäs, Electrochemically induced volume changes in poly (3, 4-ethylenedioxythiophene), Chemistry of materials 8 (10) (1996) 2439-2443.

[6] X. Lin, J. Li, E. Smela, S. Yip, Polaron-induced conformation change in single polypyrrole chain: An intrinsic actuation mechanism, International Journal of Quantum Chemistry 102 (5) (2005) 980-985.

[7] T. F. Otero, H.-J. Grande, J. Rodríguez, Reinterpretation of polypyrrole electrochemistry after consideration of conformational relaxation processes, The Journal of Physical Chemistry B 101 (19) (1997) 36883697.

[8] A. Elschner, S. Kirchmeyer, W. Lovenich, U. Merker, K. Reuter, PEDOT: principles and applications of an intrinsically conductive polymer, CRC Press, 2010.

[9] X. Wang, Understanding Actuation Mechanisms of Conjugated Polymer Actuators: Ion Transport, ProQuest, 2007.

[10] M. Gandhi, P. Murray, G. Spinks, G. Wallace, Mechanism of electromechanical actuation in polypyrrole, Synthetic Metals 73 (3) (1995) 247256.

[11] S. Skaarup, L. Bay, K. Vidanapathirana, S. Thybo, P. Tofte, K. West, Simultaneous anion and cation mobility in polypyrrole, Solid State Ionics 159 (1) (2003) 143-147.

[12] L. Bay, T. Jacobsen, S. Skaarup, K. West, Mechanism of actuation 
in conducting polymers: osmotic expansion, The Journal of Physical Chemistry B 105 (36) (2001) 8492-8497.

[13] T. Otero, I. Boyano, Comparative study of conducting polymers by the escr model, The Journal of Physical Chemistry B 107 (28) (2003) 67306738.

[14] G. A. Snook, P. Kao, A. S. Best, Conducting-polymer-based supercapacitor devices and electrodes, Journal of Power Sources 196 (1) (2011) $1-12$.

[15] A. Punning, I. Must, I. Põldsalu, V. Vunder, R. Temmer, K. Kruusamäe, F. Kaasik, J. Torop, P. Rinne, T. Lulla, Lifetime measurements of ionic electroactive polymer actuators, Journal of Intelligent Material Systems and Structures 25 (18) (2014) 2267-2275.

[16] J. D. Madden, D. Rinderknecht, P. A. Anquetil, I. W. Hunter, Creep and cycle life in polypyrrole actuators, Sensors and Actuators A: Physical 133 (1) (2007) 210-217.

[17] A. Punning, I. Must, I. Põldsalu, V. Vunder, F. Kaasik, R. Temmer, A. Aabloo, Long-term degradation of the ionic electroactive polymer actuators, in: SPIE Smart Structures and Materials+ Nondestructive Evaluation and Health Monitoring, International Society for Optics and Photonics, 2015, pp. 94300S-94300S.

[18] I. S. Romero, N. P. Bradshaw, J. D. Larson, S. Y. Severt, S. J. Roberts, M. L. Schiller, J. M. Leger, A. R. Murphy, Biocompatible electrome- 
chanical actuators composed of silk-conducting polymer composites, Advanced Functional Materials 24 (25) (2014) 3866-3873.

[19] V. Vunder, A. Punning, A. Aabloo, Long-term response of ionic electroactive polymer actuators in variable ambient conditions, in: SPIE Smart Structures and Materials+ Nondestructive Evaluation and Health Monitoring, International Society for Optics and Photonics, 2015, pp. 94300R-94300R.

[20] D. Melling, S. Wilson, E. W. Jager, The effect of film thickness on polypyrrole actuation assessed using novel non-contact strain measurements, Smart Materials and Structures 22 (10) (2013) 104021.

[21] L. Valero, J. G. Martinez, T. F. Otero, Creeping and structural effects in faradaic artificial muscles, Journal of Solid State Electrochemistry (2015) 1-7.

[22] K. Kaneto, H. Suematsu, K. Yamato, Training effect and fatigue in polypyrrole-based artificial muscles, Bioinspiration \& biomimetics 3 (3) (2008) 035005.

[23] K. Kaneto, T. Shinonome, K. Tominaga, W. Takashima, Electrochemical creeping and actuation of polypyrrole in ionic liquid, Japanese Journal of Applied Physics 50 (9R) (2011) 091601.

[24] K. Tominaga, H. Hashimoto, W. Takashima, K. Kaneto, Training and shape retention in conducting polymer artificial muscles, Smart Materials and Structures 20 (12) (2011) 124005. 
[25] X. Wang, E. Smela, Color and volume change in ppy (dbs), The Journal of Physical Chemistry C 113 (1) (2008) 359-368.

[26] E. Smela, N. Gadegaard, Surprising volume change in ppy (dbs): an atomic force microscopy study, Advanced Materials 11 (11) (1999) 953957.

[27] E. Frackowiak, F. Beguin, Carbon materials for the electrochemical storage of energy in capacitors, Carbon 39 (6) (2001) 937-950.

[28] M. Moniruzzaman, K. I. Winey, Polymer nanocomposites containing carbon nanotubes, Macromolecules 39 (16) (2006) 5194-5205.

[29] E. T. Thostenson, Z. Ren, T.-W. Chou, Advances in the science and technology of carbon nanotubes and their composites: a review, Composites science and technology 61 (13) (2001) 1899-1912.

[30] N. Khalili, H. E. Naguib, R. H. Kwon, On the geometrical and mechanical multi-aspect optimization of ppy/mwcnt actuators, in: SPIE Smart Structures and Materials+ Nondestructive Evaluation and Health Monitoring, International Society for Optics and Photonics, 2014, pp. 90561S-90561S.

[31] P. V. Pillai, Development and characterization of conducting polymer actuators, Ph.D. thesis, Massachusetts Institute of Technology (2011).

[32] M. Tahhan, V.-T. Truong, G. M. Spinks, G. G. Wallace, Carbon nanotube and polyaniline composite actuators*, Smart materials and structures 12 (4) (2003) 626. 
[33] J. Torop, A. Aabloo, E. W. Jager, Novel actuators based on polypyrrole/carbide-derived carbon hybrid materials, Carbon 80 (2014) 387-395.

[34] W. Zheng, J. M. Razal, P. G. Whitten, R. Ovalle-Robles, G. G. Wallace, R. H. Baughman, G. M. Spinks, Artificial muscles based on polypyrrole/carbon nanotube laminates, Advanced materials 23 (26) (2011) $2966-2970$.

[35] M. Endo, M. S. Strano, P. M. Ajayan, Potential applications of carbon nanotubes, in: Carbon nanotubes, Springer, 2008, pp. 13-62.

[36] Z. Spitalsky, D. Tasis, K. Papagelis, C. Galiotis, Carbon nanotubepolymer composites: chemistry, processing, mechanical and electrical properties, Progress in polymer science 35 (3) (2010) 357-401.

[37] D.-J. Yun, K. Hong, S. h. Kim, W.-M. Yun, J.-y. Jang, W.-S. Kwon, C.-E. Park, S.-W. Rhee, Multiwall carbon nanotube and poly (3, 4ethylenedioxythiophene): polystyrene sulfonate (pedot: Pss) composite films for transistor and inverter devices, ACS applied materials \& interfaces 3 (1) (2011) 43-49.

[38] J. Zhang, L. Gao, J. Sun, Y. Liu, Y. Wang, J. Wang, Incorporation of single-walled carbon nanotubes with pedot/pss in dmso for the production of transparent conducting films, Diamond and Related Materials $22(2012) 82-87$.

[39] E. Flahaut, R. Bacsa, A. Peigney, C. Laurent, Gram-scale ccvd synthe- 
sis of double-walled carbon nanotubes, Chemical Communications (12) (2003) 1442-1443.

[40] F. Seichepine, E. Flahaut, C. Vieu, A simple and versatile method for statistical analysis of the electrical properties of individual double walled carbon nanotubes, Microelectronic Engineering 88 (7) (2011) 1637-1639.

[41] A. Simaite, B. Tondu, P. Soueres, C. Bergaud, Hybrid pvdf/pvdf-graftpegma membranes for improved interface strength and lifetime of pedot: Pss/pvdf/ionic liquid actuators, ACS applied materials \& interfaces 7 (36) (2015) 19966-19977.

[42] A. Simaite, B. Tondu, F. Mathieu, P. Souéres, C. Bergaud, Simple casting based fabrication of pedot: Pss-pvdf-ionic liquid soft actuators, in: SPIE Smart Structures and Materials+ Nondestructive Evaluation and Health Monitoring, International Society for Optics and Photonics, 2015, pp. 94301E-94301E.

[43] D. A. Mengistie, P.-C. Wang, C.-W. Chu, Effect of molecular weight of additives on the conductivity of pedot: Pss and efficiency for itofree organic solar cells, Journal of Materials Chemistry A 1 (34) (2013) 9907-9915.

[44] N. Khalili, H. Naguib, R. Kwon, Electrochemomechanical constrained multiobjective optimization of ppy/mwcnt actuators, Smart Materials and Structures 23 (10) (2014) 105022.

[45] T. Sugino, K. Kiyohara, I. Takeuchi, K. Mukai, K. Asaka, Improving the 
actuating response of carbon nanotube/ionic liquid composites by the addition of conductive nanoparticles, Carbon 49 (11) (2011) 3560-3570.

[46] H. Okuzaki, S. Takagi, F. Hishiki, R. Tanigawa, Ionic liquid/polyurethane/pedot: Pss composites for electro-active polymer actuators, Sensors and Actuators B: Chemical 194 (2014) 59-63.

[47] B. Fan, Y. Xia, J. Ouyang, Novel ways to significantly enhance the conductivity of transparent pedot: Pss, in: SPIE Photonic Devices+ Applications, International Society for Optics and Photonics, 2009, pp. 74151Q-74151Q. 


\title{
Supporting Information for \\ Spray-coated carbon nanotube carpets for creeping reduction of conducting polymer based artificial muscles
}

\author{
Aiva Simaite $^{\mathrm{e}, \mathrm{f}, *}$, Aude Delagarde ${ }^{\mathrm{e}, \mathrm{g}}$, Bertrand Tondu ${ }^{\mathrm{e}, \mathrm{f}}$, Philippe Souères $^{\mathrm{e}, \mathrm{h}}$, \\ Emmanuel Flahaut ${ }^{\mathrm{e}, \mathrm{g}}$, Christian Bergaud ${ }^{\mathrm{e}, \mathrm{h}, *}$ \\ ${ }^{e}$ CNRS, LAAS, 7 avenue du Colonel Roche, F-31400, Toulouse, France \\ ${ }^{f}$ Univ de Toulouse, INSA, LAAS, F-31400, Toulouse, France \\ ${ }^{g}$ Univ de Toulouse, Institut Carnot CIRIMAT, 118 route de Narbonne, 31062, Toulouse, \\ France \\ ${ }^{h}$ Univ de Toulouse, LAAS, F-31400, Toulouse, France
}

*Corresponding author

Email addresses: asimaite@iit.edu (Aiva Simaite), delagarde@laas.fr (Aude Delagarde), tondu@insa-toulouse.fr (Bertrand Tondu), tondu@insa-toulouse.fr (Philippe Souères), flahaut@chimie.ups-tlse.fr (Emmanuel Flahaut), bergaud@laas.fr (Christian Bergaud) 


\section{S. Additional Figures}
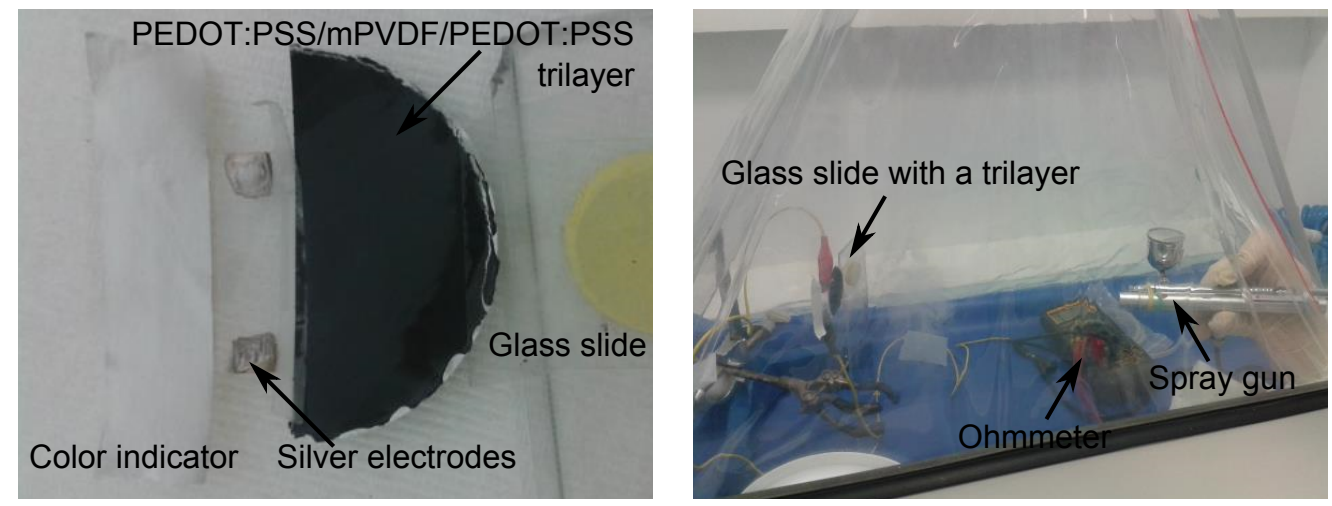

Figure S.1: Picture of PEDOT:PSS/PVDF/PEDOT:PSS trilayer attached on the glass slides next to silver electrodes and picture of the set-up used for spraying carbon nanotubes.

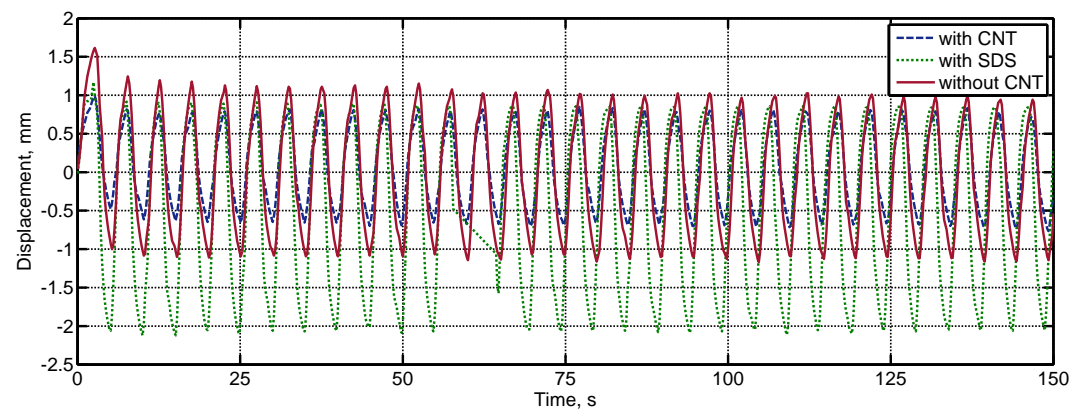

Figure S.2: Displacement profiles of trilayer actuators with and without post-treatments (lack of response of actuator with treated with SDS (green dotted line) between 50 to 75 is due to stopped video recording). $1.5 \mathrm{~V}$ square wave was used for actuation. 


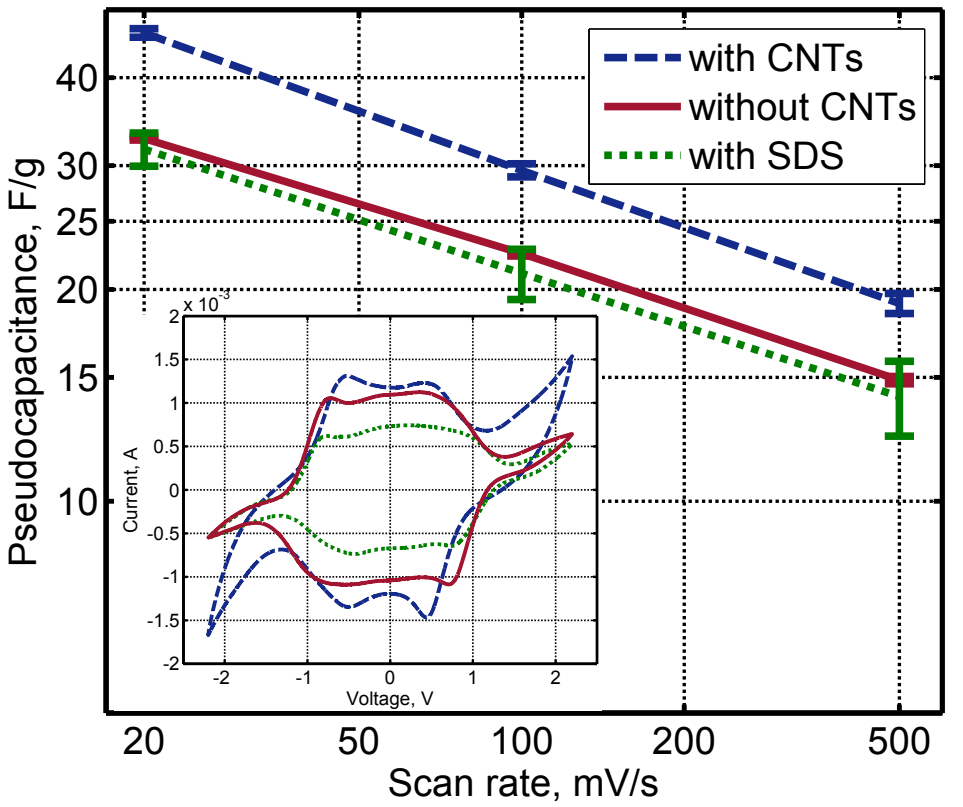

Figure S.3: Calculated specific capacitance of trialayers before and after post-treatment at different scan rates. (Inset) CV voltammogram of actuators before post-treatment (red full line) and with CNT (blue dashed line) and SDS (green dotted line) sprayed obtained with $20 \mathrm{mV} / \mathrm{s}$ scan rate. 